\title{
Improved Therapeutic Effect of Puerarin-Encapsulated PEG-PLGA Nanoparticle on an In Vitro Cerebral Infarction Model
}

\author{
Lei Li, ${ }^{1}$ Yan $\mathrm{Li}^{2}{ }^{2}$ Cheng Miao, ${ }^{1}$ and Rui Liu ${ }^{3}{ }^{3}$ \\ ${ }^{1}$ Department of Neurology, Xinxiang Central Hospital, Xinxiang 453000, Henan, China \\ ${ }^{2}$ Clinical Medical College of Tianjin Medical University, Tianjin 300270, China \\ ${ }^{3}$ Xinxiang Medical University, Xinxiang 453000, Henan, China \\ Correspondence should be addressed to Rui Liu; liurui@xxmu.edu.cn
}

Received 21 October 2019; Revised 11 December 2019; Accepted 30 December 2019; Published 28 February 2020

Guest Editor: Mingqiang Li

Copyright (c) 2020 Lei Li et al. This is an open access article distributed under the Creative Commons Attribution License, which permits unrestricted use, distribution, and reproduction in any medium, provided the original work is properly cited.

\begin{abstract}
This study was to explore the therapeutic effect and mechanism of puerarin (PUE) combined with PEGylated nanoparticles on a rat cerebral infarction cell model. In this context, PEG-PLGA/PUE nanoparticles were prepared by the thin-film hydration method, and the toxicity of PEG-PLGA/PUE nanoparticles to brain capillary endothelial cell (BCEC) was detected by MTT. The BCEC/TF cell model was obtained by induction of BCEC cells with TNF- $\alpha$. The BCEC/TF cell model was identified by immunofluorescence; the protein expression was detected by western blotting; the expression level of miR-424 in cells was measured by RT-qPCR; the targeting relationship between miR-424 and PDCD4 was confirmed by dual-luciferase reporter assay. We found that PEG-PLGA/PUE nanoparticles prepared by the thin-film hydration method had uniform particle size, regular shape, and good stability and were not toxic to cells. The vWF was widely expressed in the cytoplasm in BCECs. The BCEC/TF cell model was obtained after TNF- $\alpha$ treatment, and tissue factor (TF) was widely expressed on the cell membrane of BCEC/TF cells. Furthermore, it was observed that the PEG-PLGA/PUE nanoparticles showed better therapeutic effect on the BCEC/TF cell model than PUE. PEG-PLGA/PUE nanoparticles and PUE inhibited the expression of PDCD4 protein by increasing the expression of miR-424 in BCEC/TF cells. In summary, the therapeutic effect of PEG-PLGA/ PUE nanoparticles on the in vitro cell model of cerebral infarction is better than that of PUE. Moreover, PEG-PLGA/PUE inhibits the expression of PDCD4 protein by lowering the expression level of miR-424 in cells, thereby reducing the hazard of cerebral infarction.
\end{abstract}

\section{Introduction}

Puerarin (PUE), an isoflavone active ingredient extracted from the rhizoma of legumes Pueraria lobata and Kadsura kadsura, has antioxidant, cholesterol-lowering, and blood pressure lowering effects and is commonly used in the treatment of cardiovascular and cerebrovascular diseases [1]. However, the clinical application of PUE is limited by its low solubility, short half-life, low concentration in the brain tissue, and low absolute bioavailability in the human body.

Nano-based drug delivery systems have been used in recent years to target drugs to specific sites to improve drug bioavailability. However, ordinary nanoparticles are rapidly cleared after intravenous injection, and this clearance should be avoided during drug delivery to tissues other than the liver. A layer of hydrophilic polymer is formed by physical adsorption on the surface of ordinary nanoparticles, and such nanoparticles are called PEGylated nanoparticles. Polyethylene glycol-poly lactic-co-glycolic acid (PEGPLGA) copolymer is used as a nanopolymer carrier and has the characteristic. The PEG-PLGA polymer is amphiphilic because of having both hydrophilic and lipophilic sides. Moreover, possessing good biocompatibility and nonimmunogenicity, it has a broad application prospect in the field of PEGylated nanoparticles. Based on the above advantages, PEG-PLGA nanoparticles can therefore prolong the circulation time of the nanodrug loading system in the blood, thereby targeting specific tissues or organs and 
exerting sustained release and prolonged efficacy [2]. It has been documented [3] that PEG-PLGA nanoparticles can achieve deep penetration and have a long residence time in tumor tissues. As both the cerebral infarction site and the tumor tissue have similar inflammatory infiltration, it was speculated that PEG-PLGA nanoparticles could exert similar effects at the cerebral infarction site. Therefore, the therapeutic effect and underlying mechanism of PUE combined with PEG-PLGA nanoparticles on a rat cerebral infarction cell model were explored to provide a new theoretical basis for the treatment of cerebral infarction.

\section{Materials, Objects, and Methods}

2.1. Research and Design. This experimental protocol was designed based on an in vitro control study.

\subsection{Research Materials}

Animals. Clean SD male rats (50-60 g) were purchased from Hunan Silaike Jingda Laboratory Animal Co., Ltd.

\subsection{Drugs and Reagents}

Drugs. Puerarin (purity $\geq 98.0 \%$ ) was purchased from Shanghai Aladdin Bio-Chem Technology Co., Ltd.

Reagents. PEG-PLGA was purchased from Shanghai Shunna Biological Technology Co., Ltd.; methanol, 75\% ethanol, 4\% paraformaldehyde, and glycerol were purchased from Shandong Baiyao Chemical Co., Ltd.;MTT kit, D-Hanks, Percoll gradient centrifugation solution, BSA, and collagenase II were purchased from Beijing Kanglong Kangtai Biotechnology Co., Ltd.;polyclonal rabbit anti-human VWF antibody, rabbit anti-rat TF polyclonal antibody, and DAPI were purchased from Sangon Biological Engineering (Shanghai) Co., Ltd.;TNF- $\alpha$ was purchased from Shanghai Enzyme Lian Biological Technology Co., Ltd.; total RNA extraction kit and cDNA reverse transcription kit were purchased from Shanghai Yiyan Biological Technology Co., Ltd.; total protein extraction kit, ECL kit, and dual-luciferase reporter assay kit were purchased from Nanjing Camilo Biological Engineering Co., Ltd..

\subsection{Experimental Method}

2.4.1. Preparation of PEG-PLGA/PUE Nanoparticles. PEG-PLGA/PUE nanoparticles were prepared by the thinfilm hydration method with the following process: a certain amount of PUE and PEG-PLGA were weighed and completely dissolved in a certain volume of methanol. The solution was placed in a rotary flask at $35^{\circ} \mathrm{C}$ and protected by argon. The heating was stopped when a dry film is formed. After $10 \mathrm{~h}$ vacuuming at room temperature, the remaining organic solvent was removed and the film was hydrated by adding PBS buffer, which was followed by hydration at suitable temperature for $60 \mathrm{~min}$, ultrasonic for $20 \mathrm{~min}$, and standing at room temperature for $1 \mathrm{~h}$. Afterwards, the precipitate was removed by filtering 3 to 4 times with $0.2 \mu \mathrm{m}$ micropores to make the nanoparticles uniform in particle size, and PEG-PLGA/PU nanoparticles were obtained by being freeze-dried for $24 \mathrm{~h}$.

2.4.2. Determination of Encapsulation Efficiency and Drug Loading. The $500 \mu \mathrm{L}$ of PEG-PLGA/PUE nanoparticles after micropore filtration was centrifugated at $12000 \mathrm{rpm}$ for $10 \mathrm{~min}$, with high speed and low temperature, and the upper layer was drug-loaded nanoparticles. The encapsulation structure of the nanoparticles was destroyed by adding 10 volumes of methanol solution to completely release PUE, and the drug loading of PUE in the nanoparticles was measured by HPLC and recorded as $\mathrm{M}_{\text {-load. }}$. The same method was used to calculate the total amount of drug in $500 \mu \mathrm{L}$ of nanoparticles, recorded as $\mathrm{M}_{\text {-total, }}$, and the PUE encapsulation efficiency was worked out by using the following formula: PUE encapsulation efficiency $=\mathrm{M}_{\text {-load }} /$ $\mathrm{M}_{\text {-total. }}$

An appropriate amount of PEG-PLGA/PUE nanoparticle lyophilized powder was weighed, with its mass recorded as $\mathrm{W}_{1}$. The encapsulation structure of the nanoparticles was then destroyed by adding 10 volumes of methanol to the powder, and the amount of drug in the nanoparticles was measured by HPLC and recorded as $\mathrm{W}_{0}$. The PUE drug loading was worked out by using the following formula: PUE drug loading $=W_{0} / \mathrm{W}_{1}$.

\subsubsection{Identification of PEG-PLGA/PUE Nanoparticles.} An appropriate amount of prepared PEG-PLGA/PUE nanoparticles was diluted with distilled water and evenly distributed with the use of ultrasound. One drop of nanoparticle suspension was placed onto a copper grid with the excess liquid sucked off and dried at room temperature. The morphology of the nanomicelles was then observed under a transmission electron microscope when there was no liquid on the copper grid. The particle size distribution of the PEG-PLGA/PUE nanoparticles was determined with a zeta potentiometer (Zetasizer Nano-ZS; Malvern Instruments). An appropriate number of nanoparticles were diluted to $10 \mathrm{mg} / \mathrm{mL}$ using distilled water, and $0.5 \mathrm{~mL}$ of nanoparticle solution was added to $2.5 \mathrm{~mL}$ of sodium sulfate solution at different concentrations. The solution was allowed to stand for $10 \mathrm{~min}$ at $37^{\circ} \mathrm{C}$ followed by the measurement of the absorbance of the nanoparticles at $560 \mathrm{~nm}$ using a spectrophotometer, by which the stability of the nanoparticles was evaluated.

2.4.4. MTT Assay for Cell Viability. Cells in the logarithmic phase of growth were digested with trypsin, and single-cell suspensions with a cell density of $5 \times 10^{4}$ cells $/ \mathrm{mL}$ were adjusted. The cells were seeded in a 96-well plate at a concentration of $100 \mu \mathrm{L}$ per well, and the intervention drug was added after the cells covered the bottom of the well. Six replicate wells were set in each group, and a blank control was set. The cells were incubated for $72 \mathrm{~h}$ at $37^{\circ} \mathrm{C}, 5 \% \mathrm{CO}_{2}$. Afterwards, each well was added $10 \mu \mathrm{L}$ of $0.5 \%$ MTT solution 
followed by another 4 hours of incubation. After the blank control group was set for zero adjustment, the absorbance A at each wavelength of $570 \mathrm{~nm}$ was determined by using a microplate reader. Cell viability was calculated using the formula: cell viability $(\%)=$ A experimental group/A control group $\times 100 \%$.

2.4.5. Isolation and Identification of Rat BCEC Cells. Isolation. Three-week-old clean SD male rats were sacrificed by cervical dislocation and immersed in $75 \%$ ethanol for $3 \mathrm{~min}$. The rat brain was obtained by isolating the cortex and rinsed 3 times with ice D-Hanks after the meninges were removed. The brain tissue cut into a $1 \mathrm{~mm}^{3}$ cube was then digested with $0.1 \%$ collagenase II for $90 \mathrm{~min}$, and the supernatant was removed by centrifugation at $1000 \mathrm{rpm}$ for $20 \mathrm{~min}$ at $4{ }^{\circ} \mathrm{C}$. BSA (20\%) was added to $1 \mathrm{~mL}$ of brain tissue in the pellet. The solution was again centrifuged at $1000 \mathrm{rpm}$ for $20 \mathrm{~min}$ at $4^{\circ} \mathrm{C}$ after the precipitate was dispersed. Again $0.1 \%$ collagenase was added at $37^{\circ} \mathrm{C}$ for digestion for $60 \mathrm{~min}$, and the solution was centrifuged again with the supernatant discarded. The dispersed precipitate was then spread evenly on the Percoll gradient solution, and after centrifugation at $4^{\circ} \mathrm{C}$ for $10 \mathrm{~min}$, obvious stratification was observed. The yellowish-white microvascular fragments were aspirated, rinsed twice, and cultured with medium for microvascular endothelial cells containing MVGS at $37^{\circ} \mathrm{C}$ in $5 \% \mathrm{CO}_{2}$.

Identification. Fixation was performed using 4\% paraformaldehyde at room temperature for $20 \mathrm{~min}$ followed by three times of PBS rinsing, $5 \mathrm{~min}$ for each time. The cells were incubated with $10 \%$ goat serum for $30 \mathrm{~min}$ at $37^{\circ} \mathrm{C}$, cultured with rabbit anti-vWF polyclonal antibody $(1: 200)$ at $4^{\circ} \mathrm{C}$ overnight, and rinsed 3 times with PBS buffer for 5 min each time. The next day, the cells rinsed with PBS buffer three times for 5 min each were incubated with DAPI $(1 \mu \mathrm{g} / \mathrm{mL})$ for $10 \mathrm{~min}$ at room temperature and then rinsed with PBS buffer three times for 5 min each. The slides were finally mounted in 50\% glycerol and observed using a confocal laser scanning microscope.

\subsubsection{Construction and Identification of the BCEC/TF Model}

Construction. The rat BCECs were seeded in 6-well plates and then incubated with TNF- $\alpha(100 \mathrm{ng} / \mathrm{mL})$ after $2 \mathrm{~d}$ for $12 \mathrm{~h}$ to stimulate TF expression.

Identification. After fixation with $4 \%$ paraformaldehyde, immunofluorescence staining was performed using rabbit anti-rat TF polyclonal antibody $(1: 200)$ according to the procedure described previously. A confocal laser scanning microscope was used for observation after mounting.

2.4.7. Protein Expression Measured by Western Blotting. Total cellular proteins were extracted using a kit, transferred to a membrane after sodium dodecyl sulfate polyacrylamide gel electrophoresis, and blocked with skimmed milk powder for $1 \mathrm{~h}$. Corresponding antibodies were added, and secondary antibodies were added after the membrane was washed. Afterwards, ECL was added for color development, which was photographed using a gel imager for observation.

2.4.8. The Expression Levels of miRNAs Measured by RT$q P C R$. The cells were seeded at a density of $1 \times 10^{6} /$ well in a 6 -well plate, and after $24 \mathrm{~h}$, the culture medium was discarded before transfection. After 24 hours of transfection, the total RNA was extracted using an RNA extraction kit and the RNA was then reverse-transcribed into cDNA. The upstream primer of miR-424 was $5^{\prime}$ - CAGCAGCAATTCATGTTTTGAA-3'; the downstream primer was $5^{\prime}$-TTGTCGTC GTTAAGTACAAAAC $-3^{\prime}$; the upstream primer of internal reference U6 was $5^{\prime}$ - CTCGCTTCGGCAGCACA-3 ${ }^{\prime}$; the downstream primer was $5^{\prime}$-AACGCTTCACGAATTT GCGT $-3^{\prime}$. The reaction was performed in $10 \mu \mathrm{l}$ of $2 \times$ All-inOne qPCR Mix, $2 \mu \mathrm{l}$ universal adaptor PCR primer, $2 \mu \mathrm{l}$ Allin-One miRNA qPCR primer, $2 \mu \mathrm{l} \mathrm{cDNA}$, and $4 \mu \mathrm{l}$ ddH $2 \mathrm{O}$. Reaction conditions were as follows: $95^{\circ} \mathrm{C}, 10 \mathrm{~s} ; 95^{\circ} \mathrm{C}, 3 \mathrm{~s}$; $80^{\circ} \mathrm{C}, 20 \mathrm{~s}$; and $72^{\circ} \mathrm{C}, 20 \mathrm{~s}$ for a total of 40 cycles.

2.4.9. Dual-Luciferase Reporter Assay. Primers for miRNA and protein $\mathrm{mRNA}$ were predicted and designed and were inserted into pDCD4-wt and pDCD-mut plasmids after being identified by sequencing. After cotransfection of cells with $20 \mathrm{ng}$ of plasmid, miR-424 mimics and miR-NC plasmid, fluorescence intensity was measured using the dual-luciferase reporter assay.

2.5. Statistical Treatment. Statistical analysis was performed using SPSS 20.0. Data were compared between groups using $t$-test and among multiple groups using one-way analysis of variance, and the specific data were represented by mean\pm standard deviation.

\section{Results}

3.1. Characterization and Evaluation of PEG-PLGA/PUE Nanoparticles. The PUE encapsulation efficiency and drug loading in PEG-PLGA/PUE nanoparticles were $83.6 \%$ and 9.7\%, respectively, suggesting that PEG-PLGA/PUE nanoparticles were successfully prepared. Figure 1 (a) shows the determination of PEG-PLGA/PUE nanoparticles by transmission electron microscopy (TEM). Figure 1(b) exhibits particle size distribution of PEG-PLGA/PUE nanoparticles, and it can be seen that PEG-PLGA/PUE formed a uniform monodisperse nanosolution $(\mathrm{PDI}=0.13)$ with an average particle size of $116.4 \mathrm{~nm}$ under the influence of water-soluble vitamin E. Figure 1(c) shows the absorbance value of the nanoparticle system at $560 \mathrm{~nm}$ measured by using a spectrophotometer. When the concentration of sodium sulfate was lower than $0.45 \mathrm{~mol} / \mathrm{L}$, the absorbance value of the nanoparticle system did not change significantly, while the absorbance value of the nanoparticle system rose significantly when the concentration of sodium sulfate was greater than $0.45 \mathrm{~mol} / \mathrm{L}$. That is, the critical flocculation concentration of PEG-PLGA/PUE nanoparticles was $0.45 \mathrm{~mol} / \mathrm{L}$, 


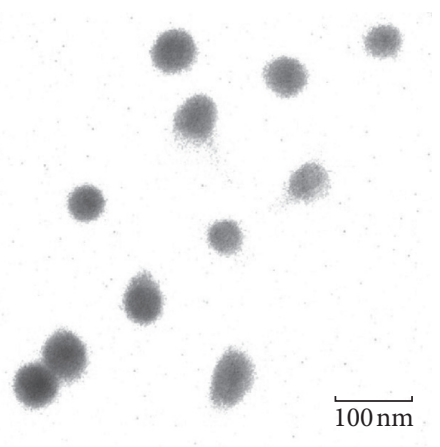

(a)

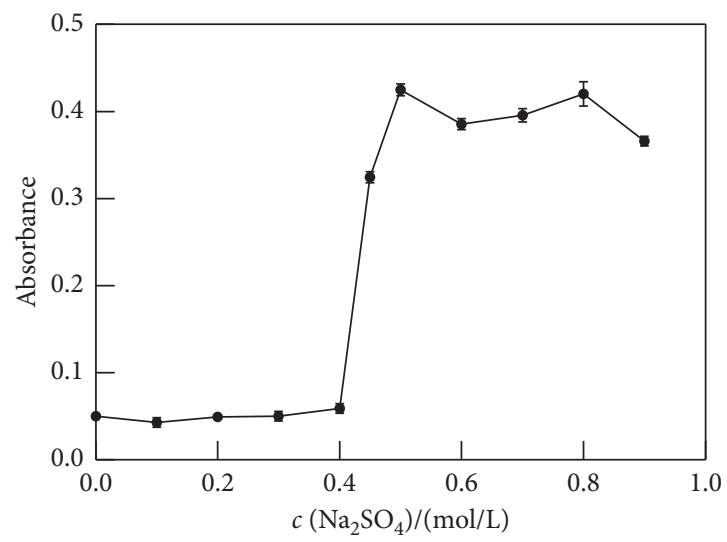

(c)

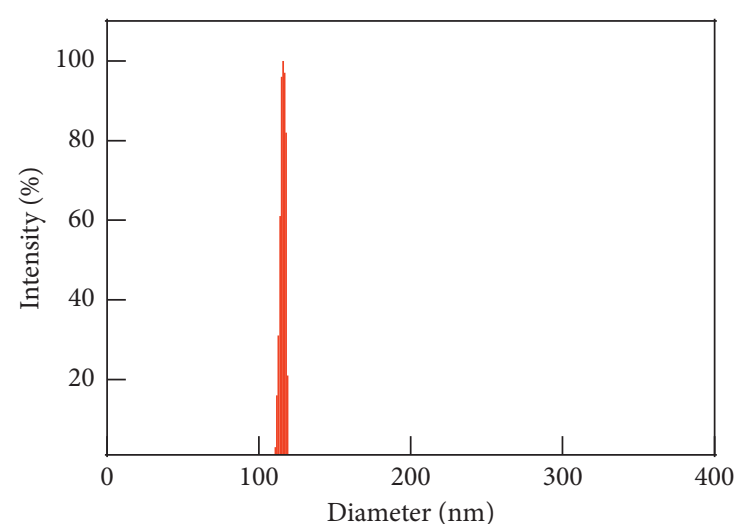

(b)

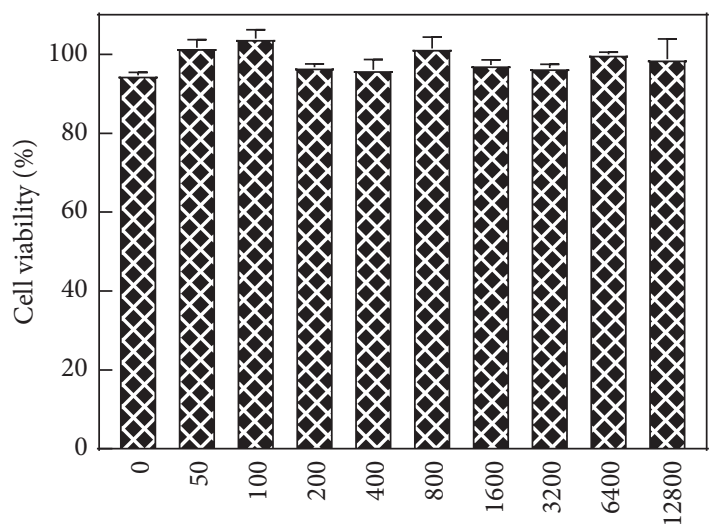

Concentration of nanoparticles $/(\mu \mathrm{g} / \mathrm{mL})$

(d)

FIgURE 1: Characterization of PEG-PLGA/PUE nanoparticles. (a, b) Size distribution and TEM image of PEG-PLGA/PUE nanoparticles. (c) Absorbance of nanoparticles. (d) Cell viability (data are shown by mean $\pm \mathrm{SD}, N=3$ ).

which was much greater than the electrolyte concentration in human blood, suggesting that PEG-PLGA/PUE nanoparticles have good stability. Figure 1(d) shows the measurement of cytotoxicity using MTT assay. After $72 \mathrm{~h}$ of coincubation of rat BCECs and PEG-PLGA/PUE nanoparticles, the survival rates of rat BCECs were all between 94.1\% and $105.6 \%$, indicating that PUE-PEG-PLGA nanoparticles had good biocompatibility.

3.2. BCEC/TF Model Construction and Identification. The product obtained by centrifugation was inoculated into a 6well plate with a medium for microvascular endothelial cells containing MVGS. After about two weeks, the basically confluent rat BCECs were in long spindle shape, forming a tight single-cell layer at the bottom of the plate, and significant contact inhibition could be observed. Rat BCECs were then subcultured. After the third-generation cells were fused, it was observed that rat BCECs showed "flagstone" appearance and the cells presented turbulence arrangement. Immunofluorescence staining results showed that rat BCECs strongly expressed vWF, an early vascular endothelial cell activation marker, mainly in the cytoplasm (Figure 2(a)). Rat BCECs were treated with TNF- $\alpha$ for $12 \mathrm{~h}$, and it was observed that TF was strongly expressed on the cell membrane of rat BCECs, while there was only trace TF expression in rat BCECs without TNF- $\alpha$ treatment (Figure 2(b)).

\subsection{Evaluation of the Effect of PEG-PLGA/PUE on BCEC/TF} Cells. Thrombomodulin (TM) and interleukin (IL-6) produce corresponding changes with the occurrence of cerebral infarction; thus, detection of the expression levels of TM and IL-6 in BCEC/TF cells by western blotting can reflect the therapeutic effect of drugs on cerebral infarction. The results of western blotting showed that the expression of TM and IL-6 in rat BCEC/TF cells of the control group was the highest, whereas that of TM and IL-6 in BCEC/TF cells cocultured with PEG-PLGA/PUE (PUE concentration of $0.5 \mathrm{mg} / \mathrm{ml})$ was the lowest $(P<0.001)$, corresponding to a lower expression of TM and IL-6 in BCEC/TF cells cocultured with PUE $(0.8 \mathrm{mg} / \mathrm{ml})(P<0.01)$ (Figure 3$)$. The above experimental results indicated that PEG-PLGA/PUE nanoparticles have better efficacy on BCEC/TF cells than PUE.

3.4. Mechanism of Action of PEG-PLGA/PUE on BCEC/TF Cells. The RT-qPCR results showed that the expression level of miR-424 in BCEC/TF cells was significantly lower than that in BCEC cells $(P<0.05)$, but the expression level of 


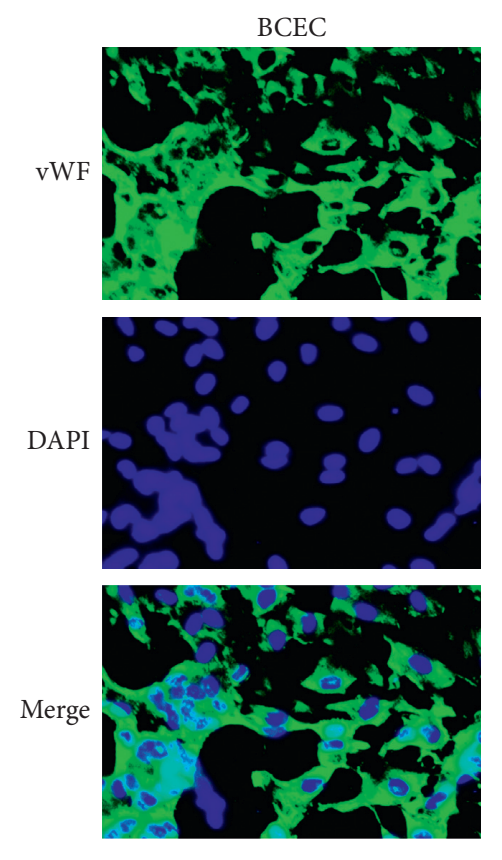

(a)

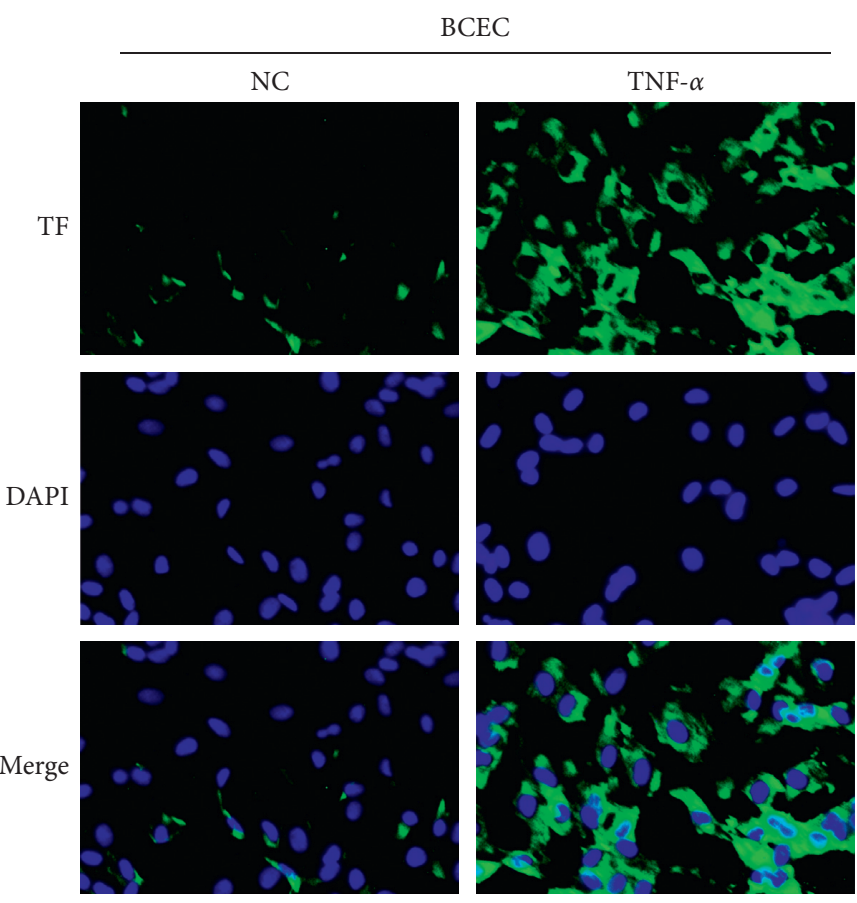

(b)

Figure 2: BCEC/TF model construction and identification. (a) The vWF expression level. (b) The TF expression level (data are shown by mean $\pm \mathrm{SD}, N=3$ ).

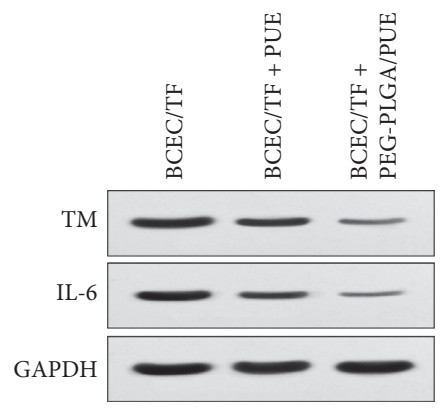

(a)

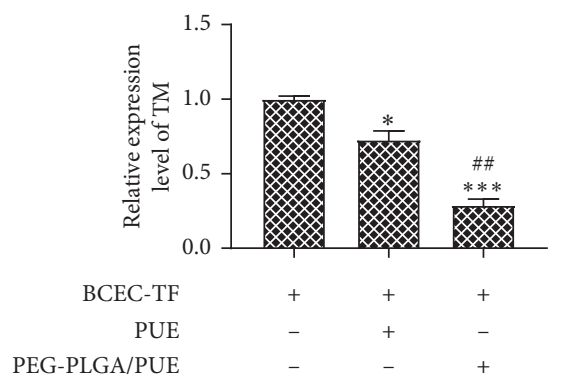

(b)

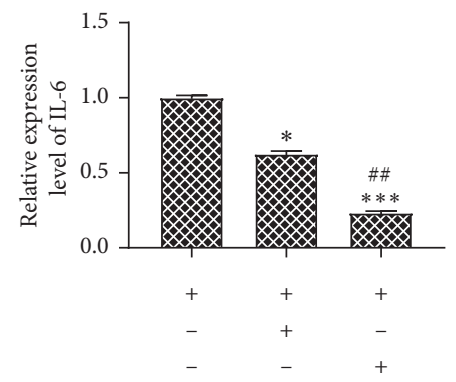

(c)

Figure 3: Expression levels of TM and IL-6 in BCEC/TF cells (data are shown by mean $\pm \mathrm{SD}, N=3 ;{ }^{*} P<0.05$ and ${ }^{* * *} P<0.001$ vs. BCEC/TF group; ${ }^{\#} P<0.01$ vs. PUE + BCEC/TF group).

miR-424 in BCEC/TF cells increased after intervention with puerarin or PEG-PLGA/PUE nanoparticles, and the difference was statistically significant $(P<0.05)$. Furthermore, the expression level of miR-424 was the highest after coculture of BCEC/TF with PEG-PLGA/PUE nanoparticles (Figure 4(a)). The miRDB database predicted that the programmed cell death factor PDCD4 might be the target gene of miR-424 (Figure 4(b)), which was confirmed by the results of the dual-luciferase reporter assay (Figure 4(c)). The western blotting results showed that the expression of programmed cell death 4 (PDCD4) was decreased in BCEC/ $\mathrm{TF}$ cells transfected with miR-424 mimics vector (Figure 4(d)). The expression of PDCD4 in BCEC/TF cells cocultured with PUE or PEG-PLGA/PUE nanoparticles was also reduced, and the difference was statistically significant $(P<0.05)$ (Figure 4(e)). In summary, PEG-PLGA/PUE nanoparticles can effectively enhance the expression of miR424 in BECE/TF cells and realize targeted inhibition of the expression of PDCD4 protein.

\section{Discussion}

Cerebral infarction is a common and frequent cerebrovascular disease that occurs at any age, and brain tissue necrosis varies with both the location and the size of thrombus. Most patients suffer massive cerebral infarction, which commonly develops in the middle cerebral artery and carotid artery. Modern pharmacological studies have shown that PUE can increase cerebral blood flow, dilate brain blood vessels, and improve brain microcirculation in patients with cerebral infarction, thereby effectively repairing the cerebral lesions [4-6]. Although some studies have shown that PUE is more 


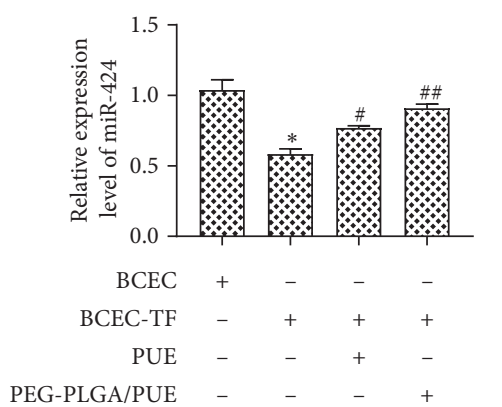

(a)

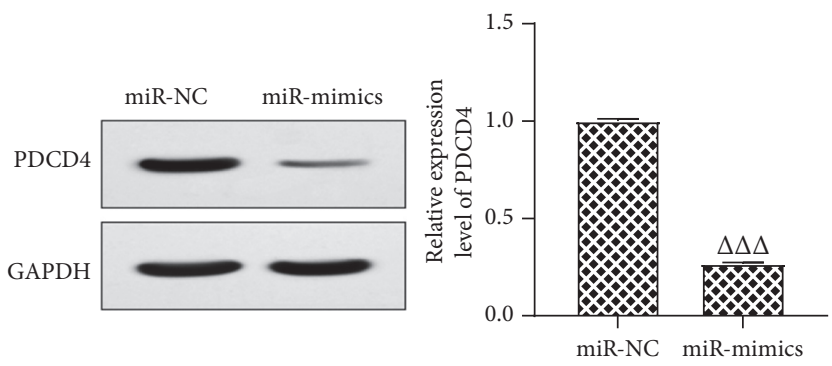

(d)

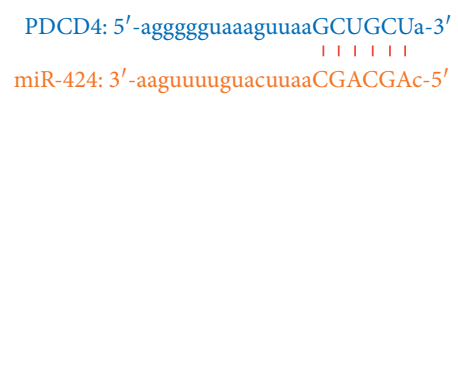

(b)
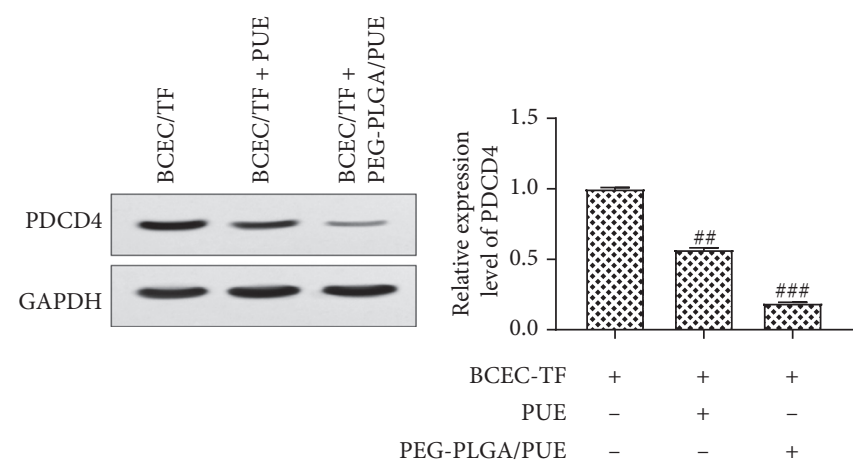

(e)

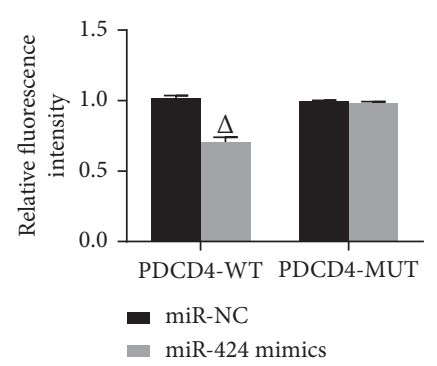

(c)

FIgUre 4: Mechanism of action of PEG-PLGA/PUE on BCEC/TF cells. (a) Relative expression of miR-424 on cells. (b) Gene sequence binding site. (c) Relative fluorescence intensity. (d, e) Protein expression level (data are shown by mean $\pm \mathrm{SD}, N=3$; ${ }^{*} P<0.05$ vs. BCEC group; ${ }^{\#} P<0.05,{ }^{\#} P<0.01$, and ${ }^{\# \# \#} P<0.001$ vs. BCEC/TF group; ${ }^{\Delta} P<0.05$ and ${ }^{\Delta \Delta} P<0.001$ vs. miR-NC group).

effective in the treatment of patients with acute cerebral infarction, its application in clinical practice is still limited due to poor solubility and extremely low bioavailability in the human body [7]. The blood-brain barrier (BBB), a unique protective structure formed by the brain during the long-term evolution, is formed in close association with brain capillary endothelial cells and prevents most substances in the blood from entering the brain, including drugs acting on brain diseases. Therefore, there is still a lack of effective drugs for some brain diseases because of the barrier that impedes the drugs with good activity from reaching the focus. Tissue factor (TF) is the cellular receptor for coagulation factor VIIa in plasma, and the TF-VIIa complex can activate FIX and FX to hydrolyze prothrombin to form thrombin, which in turn coagulates blood. TF is normally distributed outside the blood circulation, and blood cells contact TF-expressing cells to initiate the clotting process to form thrombus only in case of blood vessels and endothelial disruption. Therefore, TF with the above characteristics has become a research hotspot for brain-targeted drug delivery $[8,9]$, and the BCEC/TF cell model has been a common model for studying cerebral infarction or cerebral thrombosis in vitro. In this study, BCEC cells were obtained by isolating rat brain tissues and TF-expressing rat BCEC cells were obtained using TNF- $\alpha$ induction for experiments.

Nanoparticles, as drug carriers, can modulate drug release rate and enhance drug penetration, thus increasing drug bioavailability. However, ordinary nanoparticles are rapidly cleared after entering the human body, which cannot achieve the desired effect, so nanoparticles with "invisible" ability have attracted more and more attention from scholars. Polylactic acid modified by polyethylene glycol (PEG) makes copolymer of polylactic and polyglycolic acids (PLGA) amphiphilic, which leads to better hydrophilic nature of the nanoparticle surface, and can reduce recognition or removal by RES [10]. PEG-PLGA nanoparticles have longer circulation time in human blood after being combined with drugs, leading to the greatly improved bioavailability of drugs $[11,12]$. It was speculated that PEGPLGA/PUE nanoparticles are more effective than PUE in the cell model of cerebral infarction in vitro, and it was proved in this study that PEG-PLGA/PUE nanoparticles had a stronger inhibitory effect on the expression of TM and IL-6 proteins in rat EC/TF cells than PUE.

MicroRNAs (miRNAs), small noncoding RNA molecules about 22 nucleotides in length, are involved in a variety of physiological activities in the human body. Studies have shown that the expression intensity of miR-424 in the serum of patients with cerebral infarction is lower than that in the serum of healthy individuals and miR-424 over expression can inhibit the activation and translation of the G1/S transforming factor, which can be used as a new intervention target for cerebral infarction [13]. In this study, we found that the expression intensity of miR-424 was significantly reduced in rat BCEC/TF cells, while its expression increased again after the intervention of PUE and PEG-PLGA/PUE nanoparticles. The miRDB database prediction results showed that PDCD4 might be a downstream target gene of 
miR-424, which was confirmed by dual-luciferase reporter assay. Guo et al. reported that the reduction of PDCD4 expression could promote regeneration while inhibiting apoptosis of nerve cells, thereby bridging nerve defects in patients with cerebral infarction [14]. Furthermore, we found that the expression level of PDCD4 protein in BCEC/ TF cells was significantly higher than that in BCEC cells. The overexpression of miR-424 was followed by the decreased expression of PDCD4 protein in BCEC/TF cells.

In summary, the therapeutic effect of PEG-PLGA/PUE nanoparticles on the in vitro cell model of cerebral infarction was better than that of PUE, and PEG-PLGA/PUE reduced the hazard of cerebral infarction by increasing the expression level of miR-424 in cells while inhibiting that of PDCD4 protein.

\section{Data Availability}

The data used to support the findings of this study are included within the article.

\section{Conflicts of Interest}

The authors declare that they have no conflicts of interest.

\section{Acknowledgments}

This study was supported by grants from the Key Science and Technology Project of Henan Science and Technology Department (122102310013).

\section{References}

[1] X. S. Xie, Y. Z. Dong, D. P. Mu, X. L. Pan, and F. Y Zhang, "Evaluation on safety of puerarin injection in clinical use," Zhongguo Zhong Yao Za Zhi, vol. 43, no. 19, pp. 3956-3961, 2018.

[2] X. Y. Liu, Z. B. Jiang, J. Luo, J. H. Li, and X. B. Hu, "In vitro evaluation, cellular uptake and anti-acute myocardial ischemia effect of puerarin PEG-PLGA micelles," Zhongguo Zhong Yao Za Zhi, vol. 44, no. 11, pp. 2244-2250, 2019.

[3] Z. B. Zhao, J. Long, Y. Y. Zhao et al., "Adaptive immune cells are necessary for the enhanced therapeutic effect of sorafenibloaded nanoparticles," Biomater Sci, vol. 6, no. 4, pp. 893-900, 2018.

[4] Y. Chang, C.-Y. Hsieh, Z.-A. Peng et al., "Neuroprotective mechanisms of puerarin in middle cerebral artery occlusioninduced brain infarction in rats," Journal of Biomedical Science, vol. 16, no. 1, p. 9, 2009.

[5] H. P. Pan, J. Z. Yang, X. L. Mo et al., "Protection of puerarin on the cerebral injury in the rats with acute local ischemia," Zhongguo Zhong Yao Za Zhi, vol. 30, no. 6, pp. 457-459, 2005.

[6] R. Zhang, H. N. Guo, H. Q. Wu, H. X. Cheng, and H. Q. Wang, "Protective effect of puerarin against calcium overload after focal cerebral ischemia injury in rats," Nan Fang Yi Ke Da Xue Xue Bao, vol. 30, no. 6, pp. 1268-1271, 2010.

[7] H. T. Hu, F. Fen, and M. P. Ding, "Effects of puerarin with aspirin on the markers of damaged vascular endothelial cells in patients with acute cerebral infarction," Zhongguo Zhong Yao Za Zhi, vol. 33, no. 23, pp. 2827-2829, 2008.

[8] C. C. Visser, S. Stevanović, L. Heleen Voorwinden et al., "Validation of the transferrin receptor for drug targeting to brain capillary endothelial cells in vitro," Journal of Drug Targeting, vol. 12, no. 3, pp. 145-150, 2004.

[9] C. C. Visser, S. Stevanović, and L. Heleen Voorwinden, "Targeting liposomes with protein drugs to the blood-brain barrier in vitro," European Journal of Pharmaceutical Sciences, vol. 25, no. 2-3, pp. 299-305, 2005.

[10] K. Zhang, X. Tang, J. Zhang et al., "PEG-PLGA copolymers: their structure and structure-influenced drug delivery applications," Journal of Controlled Release, vol. 183, pp. 77-86, 2014.

[11] Z. He, J. Huang, Y. Xu et al., "Co-delivery of cisplatin and paclitaxel by folic acid conjugated amphiphilic PEG-PLGA copolymer nanoparticles for the treatment of non-small lung cancer," Oncotarget, vol. 6, no. 39, pp. 42150-42168, 2015.

[12] H. Li, Y. Tong, L. Bai et al., "Lactoferrin functionalized PEGPLGA nanoparticles of shikonin for brain targeting therapy of glioma," International Journal of Biological Macromolecules, vol. 107, no. Pt A, pp. 204-211, 2018.

[13] H. Zhao, J. Wang, L. Gao et al., "MiRNA-424 protects against permanent focal cerebral ischemia injury in mice involving suppressing microglia activation," Stroke, vol. 44, no. 6, pp. 1706-1713, 2013.

[14] Y.-B. Guo, T.-F. Ji, H.-W. Zhou, and J.-L. Yu, "Effects of microRNA-21 on nerve cell regeneration and neural function recovery in diabetes mellitus combined with cerebral infarction rats by targeting PDCD4," Molecular Neurobiology, vol. 55, no. 3, pp. 2494-2505, 2018. 